\title{
Appréciation du degré de gravité en neuropsychologie et estimation de la capacité de travail
}

\author{
Dr Vincent Verdon ${ }^{12}$ et Alexia Candal-Zürcher, M. Sc ${ }^{1}$ \\ ${ }^{1}$ Unité de neuropsychologie et logopédie, Réseau hospitalier neuchatêlois (RHNe), 2000 Neuchâtel, Suisse \\ ${ }^{2}$ CEMEDEX, Centre médical d'expertises, 1700 Fribourg, Suisse
}

\begin{abstract}
Cet article propose et présente un système d'évaluation permettant d'estimer le degré de gravité des troubles neuropsychologiques. Il englobe notamment des critères spécifiques permettant de déterminer l'intensité d'un trouble neuropsychologique et ses répercussions sur la capacité fonctionnelle et la capacité de travail. Cette nouvelle taxonomie s'appuie sur la table 8 de la Suva, destinée à évaluer les complications psychiques de lésions cérébrales en fonction de critères précis. Contrairement à la table 8 de la Suva, ce nouveau système d'évaluation est indépendant du lien de causalité établi selon la Loi sur l'assurance accident en Suisse. Cette indépendance lui permet d'être également utilisé dans d'autres contextes " assécurologiques » et pour diverses étiologies, tout en dépassant la dichotomie traditionnelle entre pathologies organiques et non organiques. Ce nouvel ensemble de critères vise aussi à améliorer la fiabilité interévaluateurs dans le processus d'évaluation de la gravité d'un trouble neuropsychologique et de sa répercussion fonctionnelle.
\end{abstract}

Mots clés : neuropsychologie, trouble, normes de référence, rendement au travail, cognition

\begin{abstract}
An assessment system for evaluating the severity of neuropsychological disorders is proposed and presented. It includes specific criteria for determining the severity of a disorder, which are in turn set in relation to the functionality and working ability of a patient or insured person. This new taxonomy is based on the criteria defined by Suva's table 8 that aims to assess psychological consequences of a brain injury. In contrast to Suva's table 8, this new assessment system is not related to any insurance context defined by the Swiss's Accident Insurance Law. It can thus be applied within the framework of other insurance contexts and for various etiologies, beyond the traditional organic and nonorganic dichotomy of pathologies. This new set of criteria shall provide a contribution towards further improvement of interrater reliability in the course of assessing the severity of neuropsychological disorders as well as assessing the functionality.
\end{abstract}

Keywords: neuropsychology, disorder, reference standards, work performance, cognition

En tant que science dédiée à l'étude des relations entre le système nerveux central et le comportement en termes de fonctionnement cognitif, affectif et social, la neuropsychologie a toujours considéré les problématiques relatives au fonctionnement d'un individu dans la vie quotidienne et dans le milieu professionnel. Toutefois, au cours des dernières années, avec la complexification des systèmes « assécurologiques » (c.-à-d., le cadre de l'assurance) et l'essor des expertises médico-légales, le neuropsychologue est de plus en plus sollicité pour statuer sur les limitations fonctionnelles au travail lors d'une évaluation standard ou dans le contexte d'une expertise multidisciplinaire pour des assurances, appréciation qui soulève de nombreux

La correspondance concernant cet article doit être adressée à / Correspondence concerning this article should be addressed to:

Vincent Verdon, spécialiste en neuropsychologie FSP et expert neuropsychologue certifié SIM.

Courriel/e-mail: vincent.verdon@rhne.ch questionnements et représente parfois un véritable défi. Dans ce contexte, cet article traite de la problématique rencontrée en neuropsychologie clinique pour attribuer un degré de gravité à un trouble neuropsychologique, pour en apprécier l'impact sur la capacité fonctionnelle et pour en estimer la capacité de travail qui en découle. L'objectif est de proposer un système d'évaluation spécifique à la neuropsychologie qui soit également indépendant du cadre assécurologique et qui soit utilisable autant dans le cadre de l'expertise neuropsychologique que lors de consultations standards (c.-à-d., hors expertise). Les systèmes actuellement existants seront présentés avant d'aborder la démarche et les propositions du groupe de travail qui a élaboré les présents critères. Plusieurs aspects importants dans le contexte de la capacité de travail (p. ex., le rôle de la neuropsychologie et l'importance de la validité des symptômes) seront ensuite discutés. 
Depuis la publication des critères de la table 8 de la Suva (2002), ces derniers sont utilisés à grande échelle, mais sans forcément toujours respecter les prérequis qui en constituent d'ailleurs les principales limitations. La table 8 de la Suva, également nommée " Atteinte à l'intégrité pour les complications psychiques des lésions cérébrales ", a pour objectif d'aider le neuropsychologue dans l'évaluation de la gravité de l'impact des troubles consécutifs à une lésion cérébrale affectant la sphère cognitive ou d'autres facultés psychiques (c.-à-d., personnalité, humeur et affect). A cette fin, elle propose une nomenclature qui précise un degré d'atteinte, allant d'une atteinte minimale de $0 \%$ à une atteinte très grave de $100 \%$, accompagnée d'une brève description de la perturbation attendue des fonctions cognitives et des autres domaines psychiques. À titre d'illustration, une atteinte moyenne, correspondant à un degré d'atteinte à l'intégrité de $50 \%$, est décrite de la façon suivante :

Nette diminution des performances d'une ou plusieurs fonctions cognitives. L'attention, la mémoire et les fonctions exécutives sont presque toujours atteintes. Des troubles peuvent cependant intéresser d'autres secteurs fonctionnels. Généralement, on observe une nette altération de la personnalité. La pulsion, l'auto-initiative, l'affect, le sens critique et le comportement social sont isolément ou de façon combinée nettement altérés. (Suva, 2002, p. 3)

Historiquement, la pratique consistait à utiliser les critères de la table 8 de la Suva pour évaluer le degré de gravité d'un trouble neuropsychologique et pour mesurer l'atteinte à l'intégrité en cas de complications psychiques de lésions cérébrales. Ces critères ont conduit à une plus grande homogénéité des conclusions lors de l'évaluation du degré de sévérité des troubles neuropsychologiques et lors de l'appréciation des capacités fonctionnelles, c'est-à-dire à une amélioration de la fiabilité interévaluateurs sur l'ensemble du territoire national suisse. La table 8 de la Suva faisait également référence dans les ouvrages traitant de l'expertise (cf. Stöckli, 2007). Depuis leur publication en 2002, les critères de la table 8 de la Suva ont également été pris en compte pour l'évaluation du degré de gravité des déficits fonctionnels neuropsychologiques consécutifs à des maladies (c.-à-d., hors du contexte de l'assuranceaccident), faute d'autres systèmes d'évaluation. Toutefois, la table 8 de la Suva connaît plusieurs limitations.
Premièrement, elle n'évalue pas le niveau d'invalidité ni le degré d'incapacité de travail (Suva, 2002). En effet, elle peut être utilisée pour estimer uniquement l'atteinte à l'intégrité en cas de troubles neuropsychologiques suite à des lésions cérébrales. Si le niveau d'atteinte à l'intégrité se définit obligatoirement au moyen de la table 8 de la Suva, il n'existe aucun système de classement concernant le niveau d'invalidité ou le degré d'incapacité de travail. Le niveau d'atteinte à l'intégrité ne correspond pas non plus nécessairement à un niveau d'invalidité ou à un degré d'incapacité de travail, ce qui prête à confusion et donne lieu à des malentendus, avec une tendance en pratique clinique à calquer le taux d'incapacité de travail sur le degré de gravité du trouble neuropsychologique de façon identique.

Un deuxième inconvénient de la table 8 de la Suva est qu'elle se rapporte exclusivement à des troubles neuropsychologiques durables et consécutifs à une atteinte cérébrale organique (c.-à-d., une lésion cérébrale structurale) causée par un accident et documentée médicalement. Sur cette base, il est aisé pour des personnes non expérimentées de conclure à tort que les déficits cognitifs n'existent qu'en cas de lésion(s) cérébrale(s) avérée(s) ou visible(s). Cependant, les déficits cognitifs peuvent également apparaître à la suite de pathologies somatiques sans lésion cérébrale visible ( $p$. ex., lors de troubles métaboliques) et sont aussi présents dans presque toutes les maladies psychiques par définition non organiques, et notamment lors de troubles affectifs ou de troubles de la personnalité (cf. American Psychiatric Association, 2015; Lautenbacher \& Gauggel, 2004). La dichotomie classique entre troubles psychiques dits organiques reposant sur un substrat physique, et troubles psychiques non organiques, sans substrat physique avéré, est ainsi problématique et peut être considérée à l'heure actuelle comme obsolète et artificielle. De plus, la distinction opérée dans la table 8 de la Suva entre les aspects cognitifs et psychiques entraînant une certaine atteinte ne propose pas de mesure objective, notamment sur le plan psychométrique, en laissant l'utilisateur apprécier de manière subjective ces aspects qui peuvent alors favoriser des différences d'appréciations selon les évaluateurs.

Une troisième limite de la table 8 de la Suva est qu'elle exige en préambule qu'une lésion organique soit la source de troubles durables, sans toutefois donner de précision sur cette durée. Or, les déficits cognitifs et autres troubles neuropsychologiques ne s'inscrivent pas obligatoirement dans la durée. Les déficits cognitifs fluctuants peuvent même constituer le symptôme majeur d'un tableau clinique (p. ex., démence à corps de Lewy) ou s'observer dans les intoxications (p. ex., prise de substance ou effets 
secondaires de médicaments). Hormis pour le lien de causalité établi par l'assurance, la nécessité d'un trouble qui s'inscrit dans la durée n'a pas lieu d'être, notamment avec la demande de documentation sur l'évolution d'un cas, une tâche importante dans la pratique clinique quotidienne des neuropsychologues. Par ailleurs, la jurisprudence évolue et les déficits cognitifs décrits par les patients avec troubles psychiques dits non organiques ne doivent plus être considérés comme des symptômes non authentiques ou surmontables (c.-à-d., surcharge indépendante de l'atteinte et pouvant être surmontés par l'individu). Cette approche visant à considérer des symptômes injustifiables de manière organique comme surmontables a été contestée par les spécialistes (Jeger, 2014), au point qu'en 2015, la Suisse a, via le tribunal fédéral, modifié sa position concernant la manière d'évaluer les patients présentant des troubles somatoformes douloureux ou d'autres affections psychosomatiques assimilées via l'ATF 9C 492/2014 du 3 juin 2015 (Frei et al., 2016). Il est toutefois important de rappeler que l'authenticité d'un tableau clinique ne doit pas être appréciée uniquement selon la preuve d'une corrélation organique des symptômes, mais aussi dans le contexte d'un examen neuropsychologique et en utilisant des protocoles permettant la validation des symptômes comme les tests de validité, les questionnaires, les critères diagnostiques (Sherman et al., 2020) ou encore les facteurs intégrés ou embedded factors (Larrabee, 2008; Lippa, 2017).

Pour répondre à l'ensemble de ces limitations, un groupe d'experts a été mandaté en 2015 par l'Association suisse des neuropsychologues (ASNP) afin d'analyser les critères existants et de proposer un système d'évaluation à la fois spécifique à la neuropsychologie et indépendant $d u$ contexte assécurologique, offrant ainsi un consensus pour tous les neuropsychologues amenés à évaluer le degré de gravité et la capacité de travail, quel que soit le contexte. Les problématiques qui ont été détaillées dans les paragraphes précédents sont à la base des objectifs de cet article et du nouveau système d'évaluation proposé (c.-à-d., la table de critères de l'ASNP) qui sont présentés dans la section suivante.

\section{Objectifs}

L'élaboration de la table de critères ASNP poursuit plusieurs objectifs :

- Aider à l'évaluation de l'incapacité de travail résultant d'un trouble neuropsychologique par le biais de valeurs indicatives sur le degré d'inaptitude au travail en adéquation avec la gravité du trouble neuropsychologique;
- Proposer un système d'évaluation indépendant du contexte de l'assurance, la table 8 de la Suva étant spécifique aux atteintes cérébrales organiques et dans le cadre de l'assurance-accident;

- Proposer des critères ( $a$ et $b$ ) qui restent compatibles avec la table 8 de la Suva en suggérant aussi un système d'évaluation conjugué aux explications relatives à son interprétation et à son utilisation;

- Améliorer la fiabilité interévaluateurs grâce à un degré plus détaillé, avec sept niveaux différents et deux critères par niveau en termes de gravité du trouble neuropsychologique, et à l'inclusion des résultats aux tests neuropsychologiques avec des valeurs standards comme critère d'appréciation;

- Répondre aux recommandations formulées dans le DSM-5 en termes d'inclusion des résultats aux tests neuropsychologiques dans l'appréciation du degré de gravité;

- Proposer des critères d'évaluation indépendants de l'étiologie des tableaux cliniques et de la dichotomie artificielle entre troubles psychiques organiques et non organiques.

Concernant les deux derniers objectifs et la compatibilité avec les classifications internationales (c.-à-d., CIM-10 et DSM-5), la table 8 de la Suva s'avère trop spécifique (cf. lésion cérébrale organique et contexte de l'assurance-accident) et ne répond pas aux recommandations du DSM-5 vis-à-vis l'inclusion des résultats aux tests neuropsychologiques dans l'appréciation du degré de gravité. Le nouveau système d'évaluation qui est proposé poursuit ainsi l'objectif d'une compatibilité avec le DSM-5 et la CIM-10, d'une part en répondant aux recommandations concernant l'inclusion des résultats aux tests (DSM-5), mais aussi en ciblant l'évaluation du degré d'atteinte indépendamment de l'étiologie (c.à-d., qu'une lésion structurale visible n'est pas indispensable) et du contexte de l'assurance, comme dans la CIM-10 notamment pour le chapitre V " Troubles mentaux et du comportement ». Contrairement à la table 8 de la Suva, la table de critères ASNP proposée peut ainsi également s'appliquer pour des déficits ne relevant pas d'une lésion structurale, et dans des situations liées à un autre contexte assécurologique que celui de l'assurance-accident.

\section{Système d'évaluation de l'incapacité de travail résultant d'un trouble neuropsychologique}

Afin de répondre aux écueils précédemment soulevés et permettre une harmonisation des pratiques helvétiques, une nouvelle nomenclature d'évaluation du degré de gravité du trouble neuropsychologique a 
été élaborée. Le Tableau 1 détaille la table de critères de l'ASNP et il résume le nouveau système d'évaluation proposé. Pour une compréhension plus aisée et en utilisant l'image du modèle de Classification internationale $\mathrm{du}$ fonctionnement $\mathrm{du}$ handicap et de la santé (CIF; Organisation mondiale de la santé, 2001), la première colonne de la table de critères de l'ASNP est plus particulièrement liée aux fonctions du modèle CIF, alors que la deuxième colonne est plus en lien avec les activités en termes de fonctionnement en vie quotidienne, sociale et professionnelle.

La première colonne de la table de critères ASNP précise deux critères diagnostiques ( $a$ et $b$ ) permettant de définir le degré de gravité du trouble neuropsychologique. Ces critères, compatibles avec les recommandations du DSM-5, distinguent d'une part des aspects psychométriques (critère $a$ ) et, d'autre part, des aspects relevant de l'affectivité, du comportement et de la personnalité (critère $b$ ). Si ces deux critères se situent souvent au même niveau d'atteinte, il peut parfois y avoir une différence notable entre l'appréciation du critère $a$ et l'appréciation du critère $b$. En effet, un patient présentant des troubles du comportement et de l'humeur au premier plan avec un fonctionnement cognitif encore relativement épargné (p. ex., dans la phase débutante d'une démence fronto-temporale) verra son degré de gravité plus en lien avec le critère $b$. De la même manière, un patient victime d'un accident vasculaire cérébral droit avec d'importants troubles attentionnels, mais sans trouble de l'humeur ou du comportement, verra son degré de gravité plus en lien avec le critère $a$. Ainsi, cette première colonne permet de mieux distinguer, mais aussi de bien prendre en compte, les aspects psychométriques d'une part (critère $a$ ) et les aspects psychiques d'autre part (critère $b$ ).

La deuxième colonne de la table de critères ASNP cible plus spécifiquement le fonctionnement dans les activités quotidiennes et professionnelles ainsi que dans l'environnement social. Elle représente une étape cruciale pour l'appréciation globale, mais aussi une continuité et une cohérence entre le degré de gravité estimé (c.-à-d., critères $a$ et $b$ en première colonne) et l'appréciation de l'incapacité de travail en pourcentages dans la troisième colonne. Dans cette deuxième colonne, le terme de capacité fonctionnelle est central et il peut se comprendre comme l'autonomie du patient dans sa capacité à réaliser des tâches quotidiennes et/ou professionnelles sur la base d'habiletés cognitives et socioaffectives. Cette colonne fournit également des indications sur la manière dont la personne est perçue par son environnement social entre la sphère privée (c.-à-d., la capacité fonctionnelle au quotidien) et la sphère professionnelle (c.-à-d., la capacité fonctionnelle dans le travail ou dans les tâches et activités requérant un niveau d'exigence élevé).

La troisième colonne de la table de critères ASNP fournit des valeurs indicatives en termes d'incapacité de travail en fonction du degré de gravité estimé et de la capacité fonctionnelle attendue. L'aspect indicatif est très important, car l'incapacité de travail est susceptible de s'écarter de ces valeurs en fonction des spécificités de la profession exercée. En effet, un trouble minime portant sur des séquelles d'aphasie chez un animateur mènera probablement à une incapacité de travail importante et bien au-dessus des valeurs indicatives (c.-à-d., de 0 à $10 \%$ ). De même, un trouble minime portant sur des séquelles de négligence spatiale unilatérale chez un chauffeur professionnel risque d'engendrer une incapacité de travail beaucoup plus importante que les valeurs indicatives proposées. L'expérience des auteurs et de leurs collègues neuropsychologues utilisant régulièrement cette table de critères ASNP depuis maintenant plus de cinq ans montre toutefois que dans la majeure partie des situations cliniques et professionnelles, l'incapacité de travail estimée sur la base des critères de gravité se situe dans les valeurs indicatives proposées en troisième colonne. À titre illustratif et sur la base de données personnelles et non publiées des auteurs, sur 40 expertises réalisées entre 2017 et 2020, la capacité de travail estimée dans l'activité habituelle se situait dans la plage de valeurs indicatives (c.-à-d., troisième colonne de la table de critères ASNP) dans plus d'un tiers des cas (c.-à-d., 25 sur 40, soit 69,4\%).

\section{Précisions sur le premier critère (critère $a$ )}

Ce critère se rapporte aux fonctions et sousfonctions cognitives qui doivent être évaluées dans le cadre de la partie psychométrique de l'examen neuropsychologique. La distinction entre fonctions ( $p$. ex., mémoire antérograde, mémoire de travail) et sousfonctions (p. ex., stockage vs récupération, charge mentale vs mise à jour) est importante pour éviter de surévaluer ou sous-évaluer le degré d'atteinte. En effet, une appréciation basée uniquement sur les fonctions, contrairement à ce que propose la table de critères ASNP, n'est pas suffisamment détaillée et risque de biaiser l'estimation (p. ex., en considérant que toute la mémoire est perturbée sans distinguer de quel processus mnésique il s'agit). La plupart des tests neuropsychologiques standardisés permettent d'évaluer une fonction via ses diverses sousfonctions . Par exemples, une épreuve de rappel libre/ indicé évalue la mémoire antérograde en distinguant les sous-fonctions de stockage (c.-à-d., rappel libre) et de récupération (c.-à-d., rappel indicé). 
Tableau 1

Critères d'évaluation du degré de gravité d'un trouble neuropsychologique et de son impact sur la capacité fonctionnelle et la capacité de travail

Degré de gravité du trouble et critères de diagnostic $(a$ et $b)$
Capacité fonctionnelle en situation de vie quotidienne et dans le travail
Valeurs indicatives sur l'incapacité de travail ${ }^{1}$

Trouble neuropsychologique minime

a) légère diminution des capacités sous l'influence d'un stress important ou décelable grâce à des tests neuropsychologiques d'une sous-fonction cognitive ou d'un nombre limité d'entre elles ( 1 à $2 \dot{E}$.- $T$. en dessous de la valeur moyenne) et/ou...

b) aucune particularité tangible ou signe uniquement présent sous l'influence d'un stress important dans les domaines de l'affectivité, du comportement ou de la personnalité

Trouble neuropsychologique léger

a) légère diminution des capacités de plusieurs sous-fonctions cognitives ( 1 à 2 É.-T. endessous de la valeur moyenne) et/ou...

b) légers signes dans les domaines de l'affectivité, du comportement ou de la personnalité

Trouble neuropsychologique léger à moyen

a) une ou au plus deux sous-fonctions cognitives sont nettement réduites (plus de $2 \dot{E}$.- $T$. endessous de la valeur moyenne) et les autres légèrement diminuées (1 à $2 E$. $-T$. en-dessous de la valeur moyenne), et/ou...

b) signes légers à moyens dans les domaines de l'affectivité, du comportement ou de la personnalité

Trouble neuropsychologique moyen

a) au moins deux sous-fonctions cognitives sont nettement réduites (plus de $2 \dot{E}$.- $T$. en dessous de la valeur moyenne) et les autres au moins légèrement diminuées ( 1 à $2 \dot{E}$.- $T$. en dessous de la valeur moyenne), et/ou...

b) signes intermédiaires dans les domaines de l'affectivité, du comportement ou de la personnalité
La personne peut se sentir perturbée de manière subjective. Sa capacité fonctionnelle n'est toutefois pas limitée dans sa vie privée au quotidien. Ses capacités professionnelles sont quasiment inaltérées. La personne ne se fait pas remarquer dans son environnement social. La capacité fonctionnelle peut toutefois être légèrement limitée lors des tâches et activités requérant un niveau d'exigences très élevé.

La capacité fonctionnelle n'est pas limitée au quotidien ni dans la plupart des sollicitations professionnelles. La personne se fait à peine remarquer dans son environnement social. La capacité fonctionnelle est toutefois limitée lors des tâches et activités requérant un niveau d'exigences élevé.

La capacité fonctionnelle est légèrement limitée au quotidien et dans la plupart des sollicitations professionnelles. La personne se fait légèrement remarquer dans son environnement social. La capacité fonctionnelle est toutefois moyennement limitée dans le travail ou lors des tâches requérant un niveau d'exigences élevé.

La capacité fonctionnelle est significativement limitée au quotidien et pour la plupart des sollicitations professionnelles. Seuls les travaux encore simples peuvent être réalisés. La personne se fait également nettement remarquer dans son environnement social. La capacité fonctionnelle est même fortement limitée dans le travail ou lors des tâches requérant un niveau d'exigences élevé.
Degré d'incapacité de travail de 0 à $10 \%$

Degré d'incapacité de travail de 10 à $30 \%$

Degré d'incapacité de travail de 30 à $50 \%$

Degré d'incapacité de travail de 50 à $70 \%$ 
Trouble neuropsychologique moyen à grave

a) la majorité des sous-fonctions cognitives est nettement diminuée (plus de $2 \dot{E}$.- $T$. en dessous de la valeur moyenne), et/ou...

b) signes moyens à graves dans les domaines de l'affectivité, du comportement ou de la personnalité

Trouble neuropsychologique grave

a) quasiment l'ensemble des fonctions cognitives est réduit de manière significative (plus de $2 \dot{E}$. $-T$. en dessous de la valeur moyenne) et ne peut absolument plus être appréhendé par des tests neuropsychologiques, et/ou...

b) signes graves dans les domaines de l'affectivité, du comportement ou de la personnalité

Trouble neuropsychologique le plus grave

Le patient réagit à peine ou souvent de manière inadaptée aux stimuli de l'environnement. Les fonctions cognitives et les autres domaines psychiques sont fortement perturbés. Les capacités cognitives ne peuvent plus être appréhendées par des tests neuropsychologiques.
La capacité fonctionnelle est significativement limitée au quotidien et pour diverses sollicitations professionnelles. Seuls les travaux encore simples peuvent être réalisés avec une étroite supervision. La personne se fait également nettement remarquer dans son environnement social. Les activités simples sont possibles, dans certaines circonstances, en atelier protégé ou dans un environnement comparable.

La capacité fonctionnelle est fortement limitée au quotidien et pour diverses sollicitations professionnelles. En outre, la personne se fait fortement remarquer dans son environnement social. La plupart des personnes concernées sont totalement inaptes au travail. Dans certaines circonstances, une activité dans un atelier protégé est encore possible.

La capacité fonctionnelle est fortement limitée au quotidien. La personne concernée est en permanence tributaire de l'aide de tiers. Une activité dans un atelier protégé n'est pas possible.
Degré d'incapacité

de travail de 70 à $90 \%$

Degré d'incapacité de travail de $100 \%$

Degré d'incapacité de travail de $100 \%$

Note. Ces valeurs indicatives ne fournissent que des pistes d'orientation. Le degré d'inaptitude au travail peut cependant s'écarter considérablement de ces valeurs indicatives en fonction des caractéristiques d'un trouble ainsi que de chaque profil d'exigences professionnelles.

Cette manière d'intégrer les résultats aux tests neuropsychologiques correspond à la procédure proposée dans le DSM-5 avec un trouble généralement caractérisé de léger pour des résultats aux tests inférieurs de un à deux écarts-types de la valeur moyenne et un trouble de gravité plus élevée lorsque les résultats aux tests sont inférieurs à la valeur moyenne de plus de deux écarts-types (American Psychological Association, 2015, page 811). Parallèlement à la procédure proposée dans le DSM-5, il est également possible d'utiliser les lignes directrices de l'ASNP proposées pour la classification et l'interprétation des résultats aux tests neuropsychologiques, dont la dernière version révisée en 2018 est également compatible avec le DSM-5. Ces lignes directrices proposent de classer les scores aux tests neuropsychologiques dans des catégories dont certaines bornes sont définies par un écart à la moyenne de 1'écart-type (c.-à-d., centile 16 et centile 84) ou de deux écarts-types (c.-à-d., centile 2 et centile 98), ce qui correspond aux écarts à la moyenne du DSM-5 pour distinguer un trouble léger d'un trouble de gravité plus élevée.

\section{Précisions sur le second critère (critère $b$ )}

Le critère $b$, quant à lui, se rapporte aux autres domaines psychiques (p. ex., affectivité, comportement social, esprit critique ou personnalité). L'appréciation des domaines psychiques complémentaires peut se faire dans le cadre d'une évaluation clinique par le biais de questionnaires d'auto-évaluation ainsi qu'au moyen de tests psychométriques standardisés. Depuis quelques années, des troubles du comportement social ou de la cognition sociale peuvent être appréciés grâce à des tests relativement bien normés comme le Faces Test (Baron-Cohen et al., 1997), le Faux-pas-Test (Stone et al., 1998) ou le Reading Mind in the Eyes Test (Bölte, 2005). Dans certains contextes, notamment en expertise ou pour des aspects spécifiques d'affectivité et de personnalité en présence d'un trouble psychiatrique majeur au premier plan, un avis psychiatrique spécialisé peut s'avérer nécessaire. Complémentaire au critère $a$, l'évaluation du critère $b$ n'en est pas moins essentielle, puisque certaines modifications non cognitives constituent parfois le signe précurseur ou persistant d'une atteinte cérébrale. Par exemple, l'évaluation de la fatigue dans la 
sclérose en plaques ou l'appréhension des modifications cognitivo-comportementales dans le cas d'un traumatisme craniocérébral sont essentielles pour évaluer le réel impact fonctionnel $\mathrm{du}$ trouble neuropsychologique.

Ainsi considérés conjointement, les critères $a$ et $b$ permettent d'aboutir à une estimation de la gravité du trouble neuropsychologique. Néanmoins, l'application formelle de ces critères diagnostiques n'est pas toujours aisée et elle peut parfois soulever de nombreuses interrogations. Les sections suivantes ont pour but de préciser certaines questions liées notamment à la sélection des tests et les modalités d'évaluation, à l'importance de la validation des symptômes, à l'évaluation de la capacité de travail, à la collaboration interdisciplinaire ainsi qu'à la question de la divergence parfois rencontrée entre la gravité des déficits cognitifs et la sévérité des symptômes dans les autres domaines psychiques, aspect qui peut parfois être difficile dans l'évaluation globale de la gravité d'un trouble.

\section{Évaluation des fonctions cognitives}

Bien que la sélection des tests neuropsychologiques soit guidée par les hypothèses du clinicien (c.-à-d., lien entre les troubles éventuellement constatés et une affection déterminée), l'examen devrait toutefois systématiquement couvrir l'ensemble des principaux domaines cognitifs. On entend par domaine une fonction cognitive donnée qui est elle-même constituée de plusieurs sous-fonctions cognitives. Par exemple, le domaine de la mémoire se divise en différentes sous-fonctions comme l'apprentissage, le stockage et la récupération. Ainsi, même si l'examen cible en premier lieu les hypothèses du clinicien en lien avec l'affection suspectée, l'omission de certaines fonctions cognitives ne permet dès lors pas d'objectiver d'éventuels troubles associés à un syndrome neuropsychologique au premier plan ou de mettre en évidence les capacités préservées dont la prise en compte est importante pour le tableau global ainsi que pour envisager une prise en charge dans un second temps. Dans un examen neuropsychologique, une sous-fonction cognitive peut être décrite à l'aide de plusieurs résultats de tests. C'est bien de l'ensemble de ces fonctions et sousfonctions cognitives dont il est question dans la première partie de la table de critères ASNP, présentée dans la section précédente. Évaluer le nombre de (sous)fonctions perturbées et la sévérité de leur dysfonctionnement permet ainsi de statuer sur le critère $a$ et, tout en pondérant avec le critère $b$, d'aider à conclure quant au degré de gravité du trouble neuropsychologique. Il convient cependant de garder à l'esprit qu'en cas de valeurs inférieures à la norme aux tests de manière isolée, il n'est pas forcément possible de conclure à la présence d'un trouble neuropsychologique compte tenu de la possibilité d'éventuelles valeurs aberrantes. En outre, chaque zone fonctionnelle doit être appréhendée si possible au moyen de plusieurs épreuves. Afin de réduire le risque de faux positifs, il convient aussi d'utiliser des données normatives disponibles afin de savoir combien de résultats inférieurs à la moyenne sont également observés chez des personnes saines de l'échantillon normatif (cf. ASNP, 2018; Colombo et al., 2016). Par exemple, l'utilisation d'une analyse de profil apporte une aide précieuse pour éviter les faux négatifs en mettant en évidence les déficits, et ce, malgré un profil en apparence normale (Crawford et al., 1998). Par ailleurs, il est aussi important que les neuropsychologues soient conscients que la probabilité d'obtenir des résultats inférieurs à la norme s'accroît au fur et à mesure que le nombre de tests réalisés augmente, et qu'en conséquence, à partir d'un certain nombre de tests effectués, des valeurs inférieures à la norme risquent d'être détectées même chez des sujets sains (Brooks et al., 2011). Enfin, lors de l'évaluation globale, il convient d'examiner la cohérence intrinsèque des résultats ainsi que leur congruence pour en faire une évaluation et une interprétation correctes.

En outre, il s'agit de garder à l'esprit qu'en fonction des exigences professionnelles, les divers domaines cognitifs revêtent des significations différentes en termes d'appréciation de la capacité fonctionnelle et de travail. Par conséquent, le choix du protocole de tests doit aussi s'orienter vers l'analyse des exigences professionnelles (Dohrenbusch et al., 2012). En principe, l'examen diagnostique approfondi des fonctions attentionnelles est d'une grande importance, celles-ci reflétant les prérequis et les bases pour d'autres fonctions cognitives (Jeger, 2011). Les fonctions exécutives et les processus de régulation émotionnelle et sociale sont également d'une importance décisive pour la réussite en matière de réinsertion professionnelle, puisqu'elles permettent l'adaptation à des situations nouvelles et l'ajustement lors des interactions sociales. Une séquence de tests plus longue peut aussi servir à éprouver l'endurance, par exemple pour évaluer l'ampleur de la fatigue (Dohrenbusch, 2007; Lukoschek et al., 2015).

Les résultats obtenus devant être vérifiables, les instruments psychodiagnostiques utilisés doivent être documentés précisément en indiquant la description $\mathrm{du}$ test, la version utilisée (p. ex., en cas de formes parallèles) et les modalités d'évaluation (c.-à-d., la correction par âge, sexe et formation). Les résultats aux tests doivent aussi être présentés sous forme de valeurs d'échelles généralement connues (p. ex., valeurs $T$, valeurs $Z$, score $Q I$ ) avant d'évaluer sur cette base le degré de gravité d'un trouble, 
conformément à la définition de la table de critères ASNP. Une représentation des résultats sous forme d'un profil est utile, car elle permet un aperçu global. L'indication des valeurs brutes est parfois contributive, notamment pour les tests sans normes reliées à la formation, à l'âge ou au sexe (p. ex., lors d'un examen des praxies ou si les valeurs divergent de telle ampleur par rapport à la norme qu'il devient impossible de les représenter à l'aide des valeurs de l'échelle). Dans le cadre de la présentation et de la description des résultats, il convient ensuite d'indiquer clairement si seules certaines sous-fonctions cognitives isolées ou la totalité d'un domaine fonctionnel présentent un déficit (p. ex., déficits en alerte tonique vs déficits des fonctions attentionnelles, comme un domaine fonctionnel cognitif global).

\section{Validité des symptômes}

Une condition préalable à l'appréciation du degré de gravité tel que proposée dans la table de critères ASNP, en particulier pour le critère $a$, réside dans la certitude que le patient ou l'assuré a réellement collaboré à l'évaluation au maximum de ses capacités et de sa motivation. Autrement dit, il s'agit de s'assurer de la validité des symptômes présentés par l'individu dans le contexte d'une pathologie déterminée. Au cours des dernières décennies, aucune autre discipline en neurosciences ne s'est enrichie d'un si grand nombre d'instruments et de protocoles de vérification de la validité avec un tel degré de pertinence (p. ex., Merten \& Dettenborn, 2009). Il existe désormais de nombreux protocoles de tests cognitifs, avec ou sans choix forcé, ainsi que des questionnaires bien normalisés pour la validation des symptômes psychiques. En outre, par l'analyse des facteurs intégrés, il devient possible d'interpréter les résultats des tests neuropsychologiques comme des paramètres de validation des troubles cognitifs (Larrabee, 2008; Lippa, 2017). Enfin, grâce aux critères de Sherman et al. (2020) pour identifier une simulation/majoration consciente (c.-à-d., malingering selon la terminologie des auteurs), ou au catalogue de critères proposés par Bianchini et ses collaborateurs (Bianchini et al., 2005; Young, 2014) pour les déficits fonctionnels simulés liés à la douleur, on dispose de systèmes d'évaluation de validation de symptômes complets qui prennent en compte non seulement les résultats aux tests, mais aussi un examen de la cohérence entre les symptômes dont se plaint le patient et sa fonctionnalité au quotidien. Dans ce cadre, il est important de garder à l'esprit que, contrairement au postulat avancé dans certains milieux psychiatriques spécialisés, l'utilisation de tests de validation des symptômes ne se réduit pas exclusivement aux troubles psychiques de nature organique (Dressing et al., 2011). En effet, la plupart des protocoles de validation des symptômes et des performances neuropsychologiques existants sont également normalisés au moyen d'échantillons de personnes expertisées présentant des troubles psychiques non organiques. Dans certaines situations en psychiatrie où des éléments de surcharge (c.-à-d., exagération) ou de simulation (c.-à-d., production volontaire de symptômes) peuvent être symptomatiques d'un trouble plus général et invalidant (p. ex., un trouble de la personnalité antisociale), la validation des symptômes psychiques (p. ex., avec le MMPI-2) joue un rôle central (Rogers, 2008). Il serait en effet peu adéquat de statuer sur le degré de gravité et encore moins sur la capacité de travail en cas de doute concernant la validité des symptômes. Même pour les tableaux de troubles psychiatriques spécifiques (p. ex., syndrome de stress post-traumatique), il existe désormais des instruments de validation de symptômes pour diagnostiquer des pathologies simulées (Young, 2014). Ces protocoles ont également été validés à l'aide d'échantillons d'assurés ayant été examinés dans le cadre d'expertises médicales (Plohmann, 2008). L'un des principaux enjeux lors de la validation des symptômes reste le choix du test approprié et la prise en compte des valeurs limites propres au trouble pour minimiser le risque de faux positifs (p. ex., chez des personnes présentant une limitation avérée des capacités intellectuelles et si aucun échantillon spécifique et qu'aucune valeur limite adaptée à cette population n'ont été utilisés pour comparaison; cf. Hurley \& Deal, 2006). En présence d'une exagération inconsciente, d'une exacerbation consciente des troubles (c.-à-d., d'une majoration délibérée des difficultés effectivement rencontrées et/ou de leurs impacts fonctionnels) ou d'une simulation intentionnelle de troubles, il n'est généralement plus possible de détecter le déficit fonctionnel cognitif de manière probante. Ainsi, comme il serait problématique de poser un diagnostic et d'appréhender des déficits dans le domaine psychopathologique en cas de description invalide des symptômes, il est crucial qu'un neuropsychologue qui justifie, suspecte ou détecte des tendances à l'exagération, une majoration ou une simulation en tienne compte lors de l'évaluation du trouble et de son impact (Dohrenbusch, 2007).

\section{Appréciation de la capacité de travail}

Lors de l'appréciation de la capacité ou de l'incapacité de travail telle que proposée dans la troisième colonne de la table de critères ASNP, le déficit doit être juridiquement pertinent par rapport à l'activité exercée jusque-là. Le trouble de la santé doit également atteindre une valeur pathologique. En d'autres termes, le trouble doit avoir pour conséquence un traitement ou une inaptitude totale ou partielle au travail (Swiss Insurance Medicine, 2013). Lors de l'évaluation, on considère en général deux aspects : la 
composante de performance (c.-à-d., sollicitation ou rendement) et la composante horaire (c.-à-d., temps de présence au poste de travail). Par exemple, une personne peut être sollicitée durant $50 \%$ du temps de travail avec une baisse de rendement de $50 \%$, et il en résulte alors une capacité de travail de $25 \%$ (c.-à-d., $50 \%$ de $50 \%$ ) ou une incapacité de $75 \%$ (c.-à-d., $100 \%$ - 25 \%; cf. Swiss Insurance Medicine, 2013). Traditionnellement, l'appréciation de l'aptitude au travail était considérée en Suisse comme une mission principalement médicale, ce qui pouvait se justifier dans le domaine de la médecine somatique, mais plus difficilement lors de l'évaluation de la capacité de travail en présence de déficits cognitifs ou psychiques. L'évolution des pratiques et le travail fourni ces dernières années par des experts neuropsychologues compétents en contexte pluridisciplinaire ont néanmoins modifié cette tradition. Grâce au développement de protocoles de tests spécifiques dont les critères de qualité ont été contrôlés (p. ex., fiabilité, validité, objectivité), la neuropsychologie dispose d'outils à la fois pertinents, spécifiques et professionnels. Bien que la validité prédictive des protocoles de tests concernant la réussite ou la réinsertion professionnelle reste sujette à débat, on peut présumer que l'évaluation des aptitudes cognitives nécessaires pour une activité déterminée, l'utilisation d'un test d'aptitude propre à la profession, l'observation du comportement dans des conditions reproduisant celles du travail ainsi que l'intégration des résultats d'une mise en situation éventuelle renforcent significativement la validité écologique (Guilmette \& Pinchot Kastner, 1996).

$\mathrm{Si}$ une incapacité de travail est fondée exclusivement ou partiellement au plan neuropsychologique, il est nécessaire de définir le degré de gravité du trouble en question et d'indiquer de quelle manière il influe sur les capacités fonctionnelles et les performances. Lors d'une expertise neuropsychologique, ainsi que parfois dans le quotidien clinique, il est également souvent nécessaire de se prononcer sur la capacité de travail. Les expertises neuropsychologiques peuvent en effet livrer des témoignages précieux et spécifiques sur le profil d'endurance et de performance actuel d'un assuré. Elles peuvent proposer des pistes thérapeutiques nécessaires à un domaine spécialisé, ainsi que des mesures permettant d'améliorer la capacité de travail et de favoriser la réinsertion. Par ailleurs, les neuropsychologues peuvent également donner des indications utiles et formuler des recommandations à l'attention des médecins responsables concernant les certificats médicaux émis. Enfin, les neuropsychologues peuvent être amenés à se prononcer sur un pronostic en termes d'évolution des troubles ou de la capacité de travail (Plohmann, 2008).
Dans ce contexte et afin de guider le clinicien dans son appréciation, les valeurs indicatives relatives à l'incapacité de travail mentionnées dans la troisième colonne de la table de critères ASNP concernent des pourcentages dans le but d'améliorer la fiabilité interévaluateurs. Le degré d'incapacité de travail peut ainsi considérablement diverger de ces valeurs indicatives en fonction des caractéristiques d'un trouble, $\mathrm{du}$ profil individuel d'exigences professionnelles ou des conditions-cadres concrètes au niveau d'un poste de travail déterminé (cf. les exemples de l'animateur et du chauffeur professionnel précédemment évoqués). Même si l'évaluation de la capacité de travail peut parfois s'écarter des valeurs indicatives données dans la table de critères ASNP, ces dernières constituent toutefois un cadre de référence important et susceptible de contribuer à améliorer la fiabilité interévaluateurs. Enfin, lors de l'appréciation de la capacité de travail, en particulier dans une activité dite adaptée, il est important de ne pas seulement prendre en compte les critères $a$ et $b$ (c.à-d., approche orientée vers la pathogenèse où l'accent est mis sur l'origine d'une affection donnée), mais aussi les ressources individuelles de la personne expertisée (c.-à-d., approche de salutogenèse orientée vers les facteurs favorisant la santé et le bien-être). Cette distinction rappelle l'importance de couvrir tous les domaines cognitifs lors de l'examen neuropsychologique, afin de mettre en évidence non seulement les fonctions atteintes, mais aussi les fonctions préservées.

\section{Degré de gravité du trouble et critères de diagnostic}

Dans certains cas, le degré de sévérité des déficits cognitifs et le degré de gravité des autres symptômes psychiques (c.-à-d., affectivité, comportement social, esprit critique, personnalité) sont susceptibles de diverger considérablement. A titre d'illustration, on sait qu'il n'est pas rare chez des patients présentant un syndrome dit dysexécutif ou un trouble organique de la personnalité consécutif à une lésion du cortex préfrontal (cf. Dilling et al., 2014 pour le code CIM10 : F07.0) de ne déceler au niveau des tests que de légers déficits, alors que ces mêmes personnes peuvent présenter des déficits marqués en termes de régulation de l'affect, de comportement social et d'esprit critique. En se référant à la table de critères ASNP et en ne prenant en compte que le critère des déficits cognitifs (critère $a$ ), ce type de profil serait probablement classé en tant que trouble neuropsychologique léger, voire minimal. Or, de sévères perturbations du comportement social (critère $b$ ) peuvent engendrer une atteinte fonctionnelle plus marquée et s'inscrire dans le cadre d'un trouble neuropsychologique moyen, voire moyen à sévère, en fonction de l'impact fonctionnel en vie quotidienne et/ 
ou professionnelle. Comme évoqué plus haut, il est important de rappeler que la prise en compte et l'évaluation des domaines psychiques d'affectivité, de comportement et de personnalité ne concernent pas que le diagnostic des troubles psychiques organiques, mais également le diagnostic des troubles psychiques non organiques. Par exemple, dans le cas de l'examen d'un trouble du spectre de l'autisme chez un individu bénéficiant d'un bon potentiel intellectuel, et donc, ne présentant pas de perturbation selon le critère $a$, seules la prise en compte et l'évaluation des déficits qualitatifs des interactions sociales et des modes de comportements stéréotypés (c.-à-d., symptômes au premier plan du tableau clinique et reflétant le critère b) permet une représentation pertinente des déficits fonctionnels et du degré d'incapacité de travail. Le clinicien se retrouve alors face à une discrépance entre la sévérité du trouble telle que définie par le critère $a$ (c.-à-d., déficits cognitifs) et celle définie par le critère $b$ (c.-à-d., symptômes psychiques). Dans ce type de situation et pour statuer sur le degré de gravité du trouble neuropsychologique, il est préconisé de retenir le critère le plus représentatif de l'incapacité fonctionnelle, en l'occurrence le critère $b$ dans l'exemple précédent, et d'estimer le degré de gravité, tout en soulignant le contraste entre la sévérité des troubles cognitifs et celle des perturbations psychiques, et en prenant soin de décrire précisément ces dernières.

\section{Distinction entre fonctions cognitives et autres domaines psychiques}

La table de critères ASNP présentée dans cet article se réfère au domaine des fonctions cognitives en intégrant également les autres domaines psychiques comme l'affectivité, le comportement social ou la personnalité, dans le but d'évaluer de la façon la plus pertinente et la plus précise possible le degré de gravité d'un trouble neuropsychologique. Il faut aussi prendre en compte la difficulté à délimiter dans certaines situations les aspects spécifiquement organiques d'un trouble psychique. Parfois, la frontière entre un trouble psychique et une composante organique per se est difficile à délimiter. C'est le cas, par exemple, des dépressions survenant après un accident vasculaire cérébral et dont les liens, notamment avec les processus physiologiques inflammatoires, sont de plus en plus documentés (cf. Spalletta et al., 2006; Pascoe et al., 2011). Dans ce contexte où la distinction entre fonctions cognitives et autres domaines psychiques est parfois malaisée, le bilan neuropsychologique pourrait se voir enrichi d'un contexte d'évaluation pluridisciplinaire avec un consensus sur l'estimation de la capacité de travail notamment en situation d'expertise. L'approche qui s'est développée ces dernières années et l'évolution des pratiques favorisent une telle collaboration interdisciplinaire. Grâce à cet échange, les tableaux cliniques psychiatriques et neuropsychologiques sont de plus en plus appréciés et discutés de manière collégiale en particulier entre les psychiatres et les neuropsychologues.

\section{Conclusion}

L'objectif de cet article était de présenter une nouvelle taxonomie pour évaluer le degré de gravité d'un trouble neuropsychologique. Rendant compte de perturbations dans les domaines cognitifs (critère $a$ ) et psychiques (critère $b$ ), la table de critères ASNP permet d'évaluer la gravité d'un trouble neuropsychologique et d'en estimer l'impact sur la capacité fonctionnelle et la capacité de travail. L'importance de prendre en compte la nature et les conditions de l'activité exercée en lien avec l'estimation du degré de gravité et de la capacité de travail a été détaillée. De telles appréciations ne peuvent toutefois pas faire l'économie de tests et autres outils visant à déterminer la validité des symptômes, condition indispensable d'une estimation fiable d'un trouble neuropsychologique et de ses conséquences. De possibles divergences entre les aspects cognitifs et psychiques ont été discutées, de même que la frontière parfois floue entre déficits cognitifs organiques et non organiques. Ce nouvel outil a pour but d'aider et de guider les cliniciens dans l'appréciation du degré de gravité d'un trouble neuropsychologique, de sorte à renforcer la fiabilité interévaluateurs. Sur le plan clinique, la table de critères ASNP offre ainsi un système de référence et un langage commun à tous les neuropsychologues qui peuvent l'utiliser et s'y référer, quelle que soit la situation clinique rencontrée. Ainsi, bien que les dispositions assécurologiques diffèrent d'un pays à l'autre, la table de critères ASNP se veut utile à l'ensemble des neuropsychologues qui rencontrent ce type de problématique en pratique clinique. En effet, facilement transposable aux autres systèmes de santé, elle pourrait permettre une plus grande harmonisation des pratiques.

\section{Conflits d'intérêts}

Les auteurs déclarent ne pas avoir de conflits d'intérêts en rapport avec cet article.

\section{Remarques}

Cet article est basé sur les recommandations d'un groupe de travail au contenu approuvé par les membres de l'assemblée générale de 2015 de l'Association suisse des neuropsychologues (ASNP), et ayant fait l'objet d'une publication en allemand (Frei et al., 2016). 


\section{Références}

American Psychiatric Association. (2015). DSM-5 : Manuel diagnostique et statistique des troubles mentaux (traduit par J.-D. Guelfi et M.-A. Crocq; $5^{\mathrm{e}}$ éd.). Elsevier Masson.

Association suisse des Neuropsychologues. (2018). Lignes directrices pour la classification et l'interprétation des résultats aux tests neuropsychologiques. Repéré à https://tinyurl.com/ reewcgd

Baron-Cohen, S., Wheelwright, S., \& Jolliffe, T. (1997). Is there a "language of the eyes"? Evidence from normal adults and adults with autism or Asperger syndrome. Visual Cognition, 4, 311-331.

Bianchini, K. J., Greve, K., W., \& Glynn, G. (2005). On the diagnosis of malingered pain-related disability: Lessons from cognitive malingering research. The Spine Journal, 5, 404-417. https:// doi.org/10.1016/j.spinee.2004.11.016

Bölte, S. (2005). Reading the Mind in the Eyes Test für Erwachsene (deutsche Fassung) von S. BaronCohen [Reading the mind in the Eyes test for adults]. J. W. Goethe Universität.

Brooks, B. L., Sherman, M. S., Iverson L. G., Slick, D. J., \& Strauss, E. (2011). Psychometric foundations for the interpretations of neuropsychological test results. In M. R. Schoenberg \& J. G. Scott (Dirs.). The Little Black Book of Neuropsychology. A Syndrome-Based Approach (pp. 893-922). New York: Springer.

Colombo, F., Amieva, H., Lecerf, T., \& Verdon V. (2016). La norme en neuropsychologie, un concept à facettes multiples. Revue de Neuropsychologie, 8 , 61-69. https://doi.org/10.3917/rne.081.0061

Crawford, J. R., Howell, D. C., \& Garthwaite, P. H. (1998). Payne and Jones revisited: Estimating the abnormality of test score differences using a modified paired samples t-test. Journal of Clinical and Experimental Neuropsychology, 20, 898-905. https://doi.org/10.1076/jcen.20.6.898.1112

Dilling, H., Mombour, W., Schmidt, M. H., \& Schulte -Markwort, E. (2014). Internationale Klassifikation psychischer Störungen. Klinisch-diagnostische Leitlinien. Hogrefe Verlag.

Dohrenbusch, R. (2007). Begutachtung somatoformer Störungen und chronifizierter Schmerzen. Konzepte, Methoden, Beispiele. Verlag W. Kohlhammer.

Dohrenbusch, R., Schneider, W., \& Merten, T. (2012). Zur bedeutung der testpsychologie bei der ICForientierten Begutachtung. In Schneider, W., Henningsen, P., Dohrenbusch, R., Freyberger, H. J., Irle, H., Köllner, V., \& Widder, B. (Dirs.). Begutachtung bei psychischen und psychosomatischen Erkrankungen: autorisierte Leitlinien und Kommentare (pp. 473-548). Verlag Hans Huber.
Dressing, H., Foerster, K., Widder, B., Schneider, F., \& Falkai, P. (2011). Zur Anwendung von Beschwerdenvalidierungstests in der psychiatrischen Begutachtung. Stellungnahme der deutschen Gesellschaft für Psychiatrie, Psychotherapie und Nervenheilkunde (DGPPN). https://doi.org/10.1055/s-0043-119801

Frei, A., Balzer, C., Gysi, F., Leros, J., Plohmann, A., \& Steiger, G. (2016). Kriterien zur bestimmung des schweregrades einer neuropsychologischen störung sowie zuordnungen zur funktions- und Arbeitsfähigkeit- Leitlinien. Zeitschrift für Neuropsychologie, 27, 107-119. https:// doi.org/10.1024/1016-264X/a000177

Guilmette, T. J. \& Pinchot Kastner, M. (1996). The prediction of vocational functioning from neuropsychological data. In R. J. Sbordone \& C. J. Long (Dirs.). Ecological Validity of Neuropsychological Nesting. St. Lucie Press.

Hurley, K. E. \& Deal, W. P. (2006). Assessment instruments measuring malingering used with individuals who have mental retardation: Potential problems and issues. Mental Retardation, 44, 112119. https://doi.org/10.1352/0047-6765(2006)44 [112:AIMMUW]2.0.CO;2

Jeger, J. (2011). Die entwicklung der « FoersterKriterien " und ihre übernahme in die bundesgerichtliche Rechtsprechung: Geschichte einer Evidenz. Repéré à https://tinyurl.com/ s3a62b2

Jeger, J. (2014). Die persönlichen ressourcen und ihre auswirkungen auf die arbeits- und wiedereingliederungsfähigkeit - eine kritische auseinandersetzung mit der überwindbarkeitspraxis. In G. Riemer-Kafka (Dir.). Psyche und Sozialversicherung, Luzerner Beiträge zur Rechtswissenschaft. Schulthess Verlag.

Larrabee, G. J. (2008). Aggregation across multiple indicators improves the detection of malingering: Relationship to likelihood ratios. The Clinical Neuropsychologist, 22, 666-679. https:// doi.org/10.1080/13854040701494987

Lautenbacher, S. \& Gauggel, S. (2004). Neuropsychologie psychischer Störungen. Springer Verlag.

Lippa, M. (2017). Performance validity testing in neuropsychology: A clinical guide, critical review, and update on a rapidly evolving literature. The Clinical Neuropsychologist, 32, 391-421. https:// doi.org/10.1080/13854046.2017.1406146

Lukoschek, C., Sterr, A., Claros-Salinas, D., Gütler, R., \& Dettmers, C. (2015). Fatigue in multiple sclerosis compared to stroke. Frontiers in Neurology, 6, 1-6. https://doi.org/10.3389/ fneur.2015.00116 
Merten, T. \& Dettenborn, H. (Dirs.). (2009). Diagnostik der Beschwerdenvalidität. Praxis der Rechtspsychologie. Deutscher Psychologen-Verlag.

Organisation mondiale de la santé. (2001). Classification internationale du fonctionnement, $d u$ handicap et de la santé. Repéré à https:// tinyurl.com $/ \mathrm{t} 7 \mathrm{fy} 47 \mathrm{v}$

Pascoe, M. C., Crewther, S. G., Carey, L. M., \& Crewther, D. P. (2011). Inflammation and depression: Why poststroke depression may be the norm and not the exception. International Journal of Stroke, 6, 128-135.

Plohmann, A. M. (2008). Relevanz neuropsychologischer gutachten zur beurteilung der arbeitsfähigkeit und integrität. Epileptologie, 25, 182-190.

Rogers, R. (2008). Clinical Assessment of Malingering and Deception. Guilford Press.

Suva. (2002). Integritätsentschädigung gemäss UVG, Tabelle 8. Repéré à https://tinyurl.com/yx7cdb99

Sherman, E. M. S., Slick, D. J., \& Iverson, G. L. (2020). Multidimensional malingering criteria for neuropsychological assessment: A 20-year update of the malingered neuropsychological dysfunction criteria. Archives of Clinical Neuropsychology, 00, 1-30.

Spalletta, G., Bossu, P., Ciaramella, A., Bria, P., Caltagirone, C., \& Robinson, R. G. (2006). The etiology of poststroke depression: A review of the literature and a new hypothesis involving inflammatory cytokines. Molecular psychiatry, 11, 984-991.

Stöckli, H. R. (2007). Besonderheiten der begutachtung in der Schweiz. In B. Widder \& P.W. Gaidzik (Dirs.), Begutachtung in der Neurologie (p. 465-476). Georg Thieme Verlag.

Stone, V. E., Baron-Cohen, S., \& Knight, R. T. (1998). Frontal lobe contributions to theory of mind. Journal of Cognitive Neuroscience, 10, 64056.

Swiss Insurance Medicine. (2013). Incapacité de travail. Lignes directrices pour l'évaluation de l'incapacité de travail par suite d'accident ou de maladie. Repéré à https://tinyurl.com/ttu7els

Young, G. (2014). Malingering, feigning, and response bias in psychiatricl psychological injury. Implications for Practice and Court. Springer. 